\title{
Viscosity approximation process for a sequence of quasinonexpansive mappings
}

\author{
Koji Aoyama ${ }^{1}$ and Fumiaki Kohsaka2*
}

"Correspondence:
f-kohsaka@oita-u.ac.jp
${ }^{2}$ Department of Computer Science
and Intelligent Systems, Oita
University, Dannoharu, Oita-shi,
Oita, 870-1192, Japan
Full list of author information is
available at the end of the article

available at the end of the article

\begin{abstract}
We study the viscosity approximation method due to Moudafi for a fixed point problem of quasinonexpansive mappings in a Hilbert space. First, we establish a strong convergence theorem for a sequence of quasinonexpansive mappings. Then we employ our result to approximate a solution of the variational inequality problem over the common fixed point set of the sequence of quasinonexpansive mappings.
\end{abstract} MSC: $47 \mathrm{H} 09 ; 47 \mathrm{H} 10 ; 41 \mathrm{~A} 65$

Keywords: viscosity approximation method; quasinonexpansive mapping; fixed point; hybrid steepest descent method

\section{Introduction}

Let $C$ be a nonempty closed convex subset of a Hilbert space. This paper is devoted to the study of strong convergence of a sequence $\left\{x_{n}\right\}$ in $C$ defined by an arbitrary point $x_{1} \in C$ and

$$
x_{n+1}=\alpha_{n} f_{n}\left(x_{n}\right)+\left(1-\alpha_{n}\right) T_{n} x_{n}
$$

for $n \in \mathbb{N}$, where $\alpha_{n}$ is a real number in $[0,1], f_{n}$ is a contraction-like mapping on $C$, and $T_{n}$ is a quasinonexpansive mapping on $C$. This iterative method (1.1) is called the viscosity approximation method [1]. In Section 3, we establish that, under some appropriate assumptions, the sequence $\left\{x_{n}\right\}$ converges strongly to a certain common fixed point of $\left\{T_{n}\right\}$ by using the technique developed in [2]. Then, in Section 4, we apply our result to approximate a solution of a variational inequality problem over the common fixed point set of $\left\{T_{n}\right\}$.

The viscosity approximation method (1.1) is based on the study of Moudafi [1], who considered a fixed point problem of a single nonexpansive mapping and proved strong convergence of sequences generated by the method. After that, $\mathrm{Xu}$ [3] extended Moudafi's results [1] in the framework of Hilbert spaces and Banach spaces; Suzuki [4] gave simple proofs of Xu's results [3]; Aoyama and Kimura [5] investigated a relationship between viscosity approximation methods and Halpern [6] type iterative methods for a sequence of nonexpansive mappings.

On the other hand, Maingé [7] adopted the viscosity approximation method for a fixed point problem of a single quasinonexpansive mapping; Wongchan and Saejung [8] extended Maingé's result [7]. Our main result (Theorem 3.1) is a generalization of Wongchan and Saejung's result [8] and is closely related to the study in [5]. Moreover, it is also appli- 
cable to an approximation method, which is called the hybrid steepest descent method [9, 10], for a variational inequality problem over the common fixed point set of a sequence of quasinonexpansive mappings.

\section{Preliminaries}

Throughout the present paper, $H$ denotes a real Hilbert space, $\langle\cdot, \cdot\rangle$ the inner product of $H,\|\cdot\|$ the norm of $H, C$ a nonempty closed convex subset of $H, I$ the identity mapping on $H, \mathbb{R}$ the set of real numbers, and $\mathbb{N}$ the set of positive integers. Strong convergence of a sequence $\left\{x_{n}\right\}$ in $H$ to $x \in H$ is denoted by $x_{n} \rightarrow x$ and weak convergence by $x_{n} \rightarrow x$.

Let $T: C \rightarrow H$ be a mapping. The set of fixed points of $T$ is denoted by Fix $(T)$. A mapping $T$ is said to be quasinonexpansive if $\operatorname{Fix}(T) \neq \emptyset$ and $\|T x-p\| \leq\|x-p\|$ for all $x \in C$ and $p \in \operatorname{Fix}(T)$; $T$ is said to be nonexpansive if $\|T x-T y\| \leq\|x-y\|$ for all $x, y \in C$; $T$ is said to be strongly quasinonexpansive if $T$ is quasinonexpansive and $T x_{n}-x_{n} \rightarrow 0$ whenever $\left\{x_{n}\right\}$ is a bounded sequence in $C$ and $\left\|x_{n}-p\right\|-\left\|T x_{n}-p\right\| \rightarrow 0$ for some point $p \in \operatorname{Fix}(T)$; $T$ is demiclosed at 0 if $T p=0$ whenever $\left\{x_{n}\right\}$ is a sequence in $C$ such that $x_{n} \rightarrow p$ and $T x_{n} \rightarrow 0$. We know that if $T: C \rightarrow H$ is quasinonexpansive, then $\operatorname{Fix}(T)$ is closed and convex; see [11, Theorem 1].

It is known that, for each $x \in H$, there exists a unique point $x_{0} \in C$ such that

$$
\left\|x-x_{0}\right\|=\min \{\|x-y\|: y \in C\} .
$$

Such a point $x_{0}$ is denoted by $P_{C}(x)$ and $P_{C}$ is called the metric projection of $H$ onto $C$. It is known that the metric projection $P_{C}$ is nonexpansive; see [12].

Let $f: C \rightarrow C$ be a mapping, $F$ a nonempty subset of $C$, and $\theta$ a real number in $[0,1)$. A mapping $f$ is said to be a $\theta$-contraction with respect to $F$ if $\|f(x)-f(z)\| \leq \theta\|x-z\|$ for all $x \in C$ and $z \in F ; f$ is said to be a $\theta$-contraction if $f$ is a $\theta$-contraction with respect to $C$. By definition, it is easy to check the following results.

Lemma 2.1 Let $F$ be a nonempty subset of $C$ and $f: C \rightarrow C$ a $\theta$-contraction with respect to $F$, where $0 \leq \theta<1$. If $F$ is closed and convex, then $P_{F} \circ f$ is a $\theta$-contraction on $F$, where $P_{F}$ is the metric projection of $H$ onto $F$.

Lemma 2.2 Let $f: C \rightarrow C$ be a $\theta$-contraction, where $0 \leq \theta<1$ and $T: C \rightarrow C$ a quasinonexpansive mapping. Then $f \circ T$ is a $\theta$-contraction with respect to $\operatorname{Fix}(T)$.

Let $D$ be a nonempty subset of $C$. A sequence $\left\{f_{n}\right\}$ of mappings of $C$ into $H$ is said to be stable on $D$ [5] if $\left\{f_{n}(z): n \in \mathbb{N}\right\}$ is a singleton for every $z \in D$. It is clear that if $\left\{f_{n}\right\}$ is stable on $D$, then $f_{n}(z)=f_{1}(z)$ for all $n \in \mathbb{N}$ and $z \in D$.

A function $\tau: \mathbb{N} \rightarrow \mathbb{N}$ is said to be eventually increasing [2] if $\lim _{n \rightarrow \infty} \tau(n)=\infty$ and $\tau(n) \leq \tau(n+1)$ for all $n \in \mathbb{N}$. By definition, we easily obtain the following.

Lemma 2.3 Let $\tau: \mathbb{N} \rightarrow \mathbb{N}$ be an eventually increasing function and $\left\{\xi_{n}\right\}$ a sequence of real numbers such that $\xi_{n} \rightarrow \xi$. Then $\xi_{\tau(n)} \rightarrow \xi$.

The following is a direct consequence of [13, Lemma 3.1].

Lemma 2.4 ([2, Lemma 3.4]) Let $\left\{\xi_{n}\right\}$ be a sequence of nonnegative real numbers which is not convergent. Then there exist $N \in \mathbb{N}$ and an eventually increasing function $\tau: \mathbb{N} \rightarrow \mathbb{N}$ such that $\xi_{\tau(n)} \leq \xi_{\tau(n)+1}$ for all $n \in \mathbb{N}$ and $\xi_{n} \leq \xi_{\tau(n)+1}$ for all $n \geq N$. 
Under the assumptions of Lemma 2.4, we cannot choose a strictly increasing function $\tau$; see [2, Example 3.3].

Let $\left\{T_{n}\right\}$ be a sequence of mappings of $C$ into $H$ such that $F=\bigcap_{n=1}^{\infty} \operatorname{Fix}\left(T_{n}\right)$ is nonempty. Then

- $\left\{T_{n}\right\}$ is said to be strongly quasinonexpansive type if each $T_{n}$ is quasinonexpansive and $T_{n} x_{n}-x_{n} \rightarrow 0$ whenever $\left\{x_{n}\right\}$ is a bounded sequence in $C$ and

$$
\left\|x_{n}-p\right\|-\left\|T_{n} x_{n}-p\right\| \rightarrow 0
$$

for some point $p \in F$;

- $\left\{T_{n}\right\}$ is said to satisfy the condition (Z) $[2,14-16]$ if every weak cluster point of $\left\{x_{n}\right\}$ belongs to $F$ whenever $\left\{x_{n}\right\}$ is a bounded sequence in $C$ such that $T_{n} x_{n}-x_{n} \rightarrow 0$.

Remark 2.5 Since $\beta_{n}-\alpha_{n} \rightarrow 0$ if and only if $\beta_{n}^{2}-\alpha_{n}^{2} \rightarrow 0$ for all bounded sequences $\left\{\alpha_{n}\right\}$ and $\left\{\beta_{n}\right\}$ in $[0, \infty),\left\{T_{n}\right\}$ is strongly quasinonexpansive type if and only if it is a strongly relatively nonexpansive sequence in the sense of $[2,17]$. See also $[18,19]$.

We know several examples of strongly quasinonexpansive type sequences satisfying the condition (Z); see [17] and Example 4.5 in Section 4.

The following lemma follows from [2, Lemma 3.5] and Remark 2.5.

Lemma 2.6 Let $\left\{T_{n}\right\}$ be a sequence of mappings of $C$ into $H$ such that $F=\bigcap_{n=1}^{\infty} \operatorname{Fix}\left(T_{n}\right)$ is nonempty, $\tau: \mathbb{N} \rightarrow \mathbb{N}$ an eventually increasing function, and $\left\{z_{n}\right\}$ a bounded sequence in $C$ such that $\left\|z_{n}-p\right\|-\left\|T_{\tau(n)} z_{n}-p\right\| \rightarrow 0$ for some $p \in F$. If $\left\{T_{n}\right\}$ is strongly quasinonexpansive type, then $T_{\tau(n)} z_{n}-z_{n} \rightarrow 0$.

In order to prove our main result in Section 3, we need the following lemmas.

Lemma 2.7 ([2, Lemma 3.6]) Let $\left\{T_{n}\right\}$ be a sequence of mappings of $C$ into $H$ such that $F=\bigcap_{n=1}^{\infty} \operatorname{Fix}\left(T_{n}\right)$ is nonempty, $\tau: \mathbb{N} \rightarrow \mathbb{N}$ an eventually increasing function, and $\left\{z_{n}\right\}$ a bounded sequence in $C$ such that $T_{\tau(n)} z_{n}-z_{n} \rightarrow 0$. Suppose that $\left\{T_{n}\right\}$ satisfies the condition (Z). Then every weak cluster point of $\left\{z_{n}\right\}$ belongs to $F$.

Lemma 2.8 ([2, Lemma 3.7]) Let $\left\{T_{n}\right\}$ be a sequence of mappings of $C$ into $H$, $F$ a nonempty closed convex subset of $H,\left\{z_{n}\right\}$ a bounded sequence in $C$ such that $T_{n} z_{n}-z_{n} \rightarrow 0$, and $u \in H$. Suppose that every weak cluster point of $\left\{z_{n}\right\}$ belongs to $F$. Then

$$
\limsup _{n \rightarrow \infty}\left\langle T_{n} z_{n}-w, u-w\right\rangle \leq 0,
$$

where $w=P_{F}(u)$.

The following lemma is well known; see [20, 21].

Lemma 2.9 Let $\left\{\xi_{n}\right\}$ be a sequence of nonnegative real numbers, $\left\{\delta_{n}\right\}$ a sequence of real numbers, and $\left\{\beta_{n}\right\}$ a sequence in $[0,1]$. Suppose that $\xi_{n+1} \leq\left(1-\beta_{n}\right) \xi_{n}+\beta_{n} \delta_{n}$ for every $n \in \mathbb{N}$, $\lim \sup _{n \rightarrow \infty} \delta_{n} \leq 0$, and $\sum_{n=1}^{\infty} \beta_{n}=\infty$. Then $\xi_{n} \rightarrow 0$. 


\section{Strong convergence of a viscosity approximation process}

In this section, we prove the following strong convergence theorem.

Theorem 3.1 Let $H$ be a real Hilbert space, $C$ a nonempty closed convex subset of $H,\left\{S_{n}\right\}$ a sequence of mappings of $C$ into $C$ such that $F=\bigcap_{n=1}^{\infty} \operatorname{Fix}\left(S_{n}\right)$ is nonempty, $\left\{\alpha_{n}\right\}$ a sequence in $(0,1]$ such that $\alpha_{n} \rightarrow 0$ and $\sum_{n=1}^{\infty} \alpha_{n}=\infty$, and $\left\{f_{n}\right\}$ a sequence of mappings of $C$ into $C$ such that each $f_{n}$ is a $\theta$-contraction with respect to $F$ and $\left\{f_{n}\right\}$ is stable on $F$, where $0 \leq \theta<1$. Let $\left\{x_{n}\right\}$ be a sequence defined by $x_{1} \in C$ and

$$
x_{n+1}=\alpha_{n} f_{n}\left(x_{n}\right)+\left(1-\alpha_{n}\right) S_{n} x_{n}
$$

for $n \in \mathbb{N}$. Suppose that $\left\{S_{n}\right\}$ is strongly quasinonexpansive type and satisfies the condition $(\mathrm{Z})$. Then $\left\{x_{n}\right\}$ converges strongly to $w \in F$, where $w$ is the unique fixed point of a contraction $P_{F} \circ f_{1}$.

Note that Lemma 2.1 implies that $P_{F} \circ f_{1}$ is a $\theta$-contraction on $F$ and hence it has a unique fixed point on $F$.

First, we show some lemmas; then we prove Theorem 3.1. In the rest of this section, we set

$$
\beta_{n}=\alpha_{n}\left(1+(1-2 \theta)\left(1-\alpha_{n}\right)\right)
$$

and

$$
\gamma_{n}=\alpha_{n}^{2}\left\|f_{n}\left(x_{n}\right)-w\right\|^{2}+2 \alpha_{n}\left(1-\alpha_{n}\right)\left\langle S_{n} x_{n}-w, f_{1}(w)-w\right\rangle
$$

for $n \in \mathbb{N}$.

Lemma 3.2 $\left\{x_{n}\right\},\left\{S_{n} x_{n}\right\}$, and $\left\{f_{n}\left(x_{n}\right)\right\}$ are bounded, and moreover,

$$
\left\|x_{n+1}-w\right\| \leq \alpha_{n}\left\|f_{n}\left(x_{n}\right)-w\right\|+\left\|S_{n} x_{n}-w\right\|
$$

and

$$
\left\|x_{n+1}-w\right\|^{2} \leq\left(1-\beta_{n}\right)\left\|x_{n}-w\right\|^{2}+\gamma_{n}
$$

hold for every $n \in \mathbb{N}$.

Proof Since $f_{n}$ is a $\theta$-contraction with respect to $F, S_{n}$ is quasinonexpansive, $w \in F \subset$ Fix $\left(S_{n}\right)$, and $\left\{f_{n}\right\}$ is stable on $F$, it follows that

$$
\begin{aligned}
& \left\|x_{n+1}-w\right\| \\
& \quad \leq \alpha_{n}\left\|f_{n}\left(x_{n}\right)-w\right\|+\left(1-\alpha_{n}\right)\left\|S_{n} x_{n}-w\right\| \\
& \quad \leq \alpha_{n}\left(\left\|f_{n}\left(x_{n}\right)-f_{n}(w)\right\|+\left\|f_{n}(w)-w\right\|\right)+\left(1-\alpha_{n}\right)\left\|S_{n} x_{n}-w\right\| \\
& \quad \leq\left(1-\alpha_{n}(1-\theta)\right)\left\|x_{n}-w\right\|+\alpha_{n}(1-\theta) \frac{\left\|f_{1}(w)-w\right\|}{1-\theta}
\end{aligned}
$$


for every $n \in \mathbb{N}$. Thus, by induction on $n$, we have

$$
\left\|S_{n} x_{n}-w\right\| \leq\left\|x_{n}-w\right\| \leq \max \left\{\left\|x_{1}-w\right\|,\left\|f_{1}(w)-w\right\| /(1-\theta)\right\} .
$$

Therefore, it turns out that $\left\{x_{n}\right\}$ and $\left\{S_{n} x_{n}\right\}$ are bounded, and moreover, $\left\{f_{n}\left(x_{n}\right)\right\}$ is also bounded.

Equation (3.2) follows from (3.4).

Next, we show (3.3). By assumption, it follows that

$$
\begin{aligned}
\left\langle S_{n} x_{n}-w, f_{n}\left(x_{n}\right)-w\right\rangle & \leq\left\|S_{n} x_{n}-w\right\|\left\|f_{n}\left(x_{n}\right)-f_{n}(w)\right\|+\left\langle S_{n} x_{n}-w, f_{n}(w)-w\right\rangle \\
& \leq \theta\left\|x_{n}-w\right\|^{2}+\left\langle S_{n} x_{n}-w, f_{1}(w)-w\right\rangle,
\end{aligned}
$$

and thus

$$
\begin{aligned}
\left\|x_{n+1}-w\right\|^{2}= & \alpha_{n}^{2}\left\|f_{n}\left(x_{n}\right)-w\right\|^{2}+\left(1-\alpha_{n}\right)^{2}\left\|S_{n} x_{n}-w\right\|^{2} \\
& +2 \alpha_{n}\left(1-\alpha_{n}\right)\left(S_{n} x_{n}-w, f_{n}\left(x_{n}\right)-w\right\rangle \\
\leq & \alpha_{n}^{2}\left\|f_{n}\left(x_{n}\right)-w\right\|^{2}+\left(\left(1-\alpha_{n}\right)^{2}+2 \alpha_{n}\left(1-\alpha_{n}\right) \theta\right)\left\|x_{n}-w\right\|^{2} \\
& +2 \alpha_{n}\left(1-\alpha_{n}\right)\left(S_{n} x_{n}-w, f_{1}(w)-w\right\rangle \\
= & \left(1-\beta_{n}\right)\left\|x_{n}-w\right\|^{2}+\gamma_{n}
\end{aligned}
$$

for every $n \in \mathbb{N}$. Therefore, (3.3) holds.

Lemma 3.3 The following hold:

- $0<\beta_{n} \leq 1$ for every $n \in \mathbb{N}$;

- $2 \alpha_{n}\left(1-\alpha_{n}\right) / \beta_{n} \rightarrow 1 /(1-\theta)$;

- $\alpha_{n}^{2}\left\|f_{n}\left(x_{n}\right)-w\right\|^{2} / \beta_{n} \rightarrow 0$

- $\sum_{n=1}^{\infty} \beta_{n}=\infty$.

Proof Since $0<\alpha_{n} \leq 1$ and $-1<1-2 \theta \leq 1$, we know that

$$
0<\alpha_{n}^{2}=\alpha_{n}\left(1+(-1)\left(1-\alpha_{n}\right)\right) \leq \beta_{n} \leq \alpha_{n}\left(1+\left(1-\alpha_{n}\right)\right)=\alpha_{n}\left(2-\alpha_{n}\right) \leq 1 .
$$

It follows from $\alpha_{n} \rightarrow 0$ that $2 \alpha_{n}\left(1-\alpha_{n}\right) / \beta_{n} \rightarrow 1 /(1-\theta)$.

Since $\left\{f_{n}\left(x_{n}\right)\right\}$ is bounded by Lemma 3.2 and

$$
\frac{\alpha_{n}^{2}}{\beta_{n}}=\frac{\alpha_{n}}{1+(1-2 \theta)\left(1-\alpha_{n}\right)} \rightarrow 0
$$

it follows that $\alpha_{n}^{2}\left\|f_{n}\left(x_{n}\right)-w\right\|^{2} / \beta_{n} \rightarrow 0$.

Finally, we prove $\sum_{n=1}^{\infty} \beta_{n}=\infty$. Suppose that $1-2 \theta \geq 0$. Then it is clear that $\beta_{n} \geq \alpha_{n}$ for every $n \in \mathbb{N}$. Thus, $\sum_{n=1}^{\infty} \beta_{n} \geq \sum_{n=1}^{\infty} \alpha_{n}=\infty$. Next, we suppose that $1-2 \theta<0$. Then it is clear that $\beta_{n}>2(1-\theta) \alpha_{n}$ for every $n \in \mathbb{N}$. Thus, $\sum_{n=1}^{\infty} \beta_{n} \geq 2(1-\theta) \sum_{n=1}^{\infty} \alpha_{n}=\infty$. This completes the proof.

Lemma 3.4 $\left\{\left\|x_{n}-w\right\|\right\}$ is convergent. 
Proof We assume, in order to obtain a contraction, that $\left\{\left\|x_{n}-w\right\|\right\}$ is not convergent. Then Lemma 2.4 implies that there exist $N \in \mathbb{N}$ and an eventually increasing function $\tau: \mathbb{N} \rightarrow \mathbb{N}$ such that

$$
\left\|x_{\tau(n)}-w\right\| \leq\left\|x_{\tau(n)+1}-w\right\|
$$

for every $n \in \mathbb{N}$ and

$$
\left\|x_{n}-w\right\| \leq\left\|x_{\tau(n)+1}-w\right\|
$$

for every $n \geq N$.

We show that $S_{\tau(n)} x_{\tau(n)}-x_{\tau(n)} \rightarrow 0$. Since $S_{\tau(n)}$ is quasinonexpansive and $w \in F \subset$ $\operatorname{Fix}\left(S_{\tau(n)}\right)$, it follows from (3.6), (3.2), and Lemmas 2.3 and 3.2 that

$$
\begin{aligned}
0 & \leq\left\|x_{\tau(n)}-w\right\|-\left\|S_{\tau(n)} x_{\tau(n)}-w\right\| \\
& \leq\left\|x_{\tau(n)+1}-w\right\|-\left\|S_{\tau(n)} x_{\tau(n)}-w\right\| \\
& \leq \alpha_{\tau(n)}\left\|f_{\tau(n)}\left(x_{\tau(n)}\right)-w\right\| \rightarrow 0
\end{aligned}
$$

as $n \rightarrow \infty$. Since $\left\{x_{\tau(n)}\right\}$ is bounded and $\left\{S_{n}\right\}$ is strongly quasinonexpansive type, Lemma 2.6 implies that $S_{\tau(n)} x_{\tau(n)}-x_{\tau(n)} \rightarrow 0$.

Since $\left\{S_{n}\right\}$ satisfies the condition (Z), it follows from Lemma 2.7 that every weak cluster point of $\left\{x_{\tau(n)}\right\}$ belongs to $F$. Thus Lemma 2.8 shows that

$$
\limsup _{n \rightarrow \infty}\left\langle S_{\tau(n)} x_{\tau(n)}-w, f_{1}(w)-w\right\rangle \leq 0
$$

Moreover, Lemmas 2.3 and 3.3 imply that $\alpha_{\tau(n)}^{2}\left\|f_{\tau(n)}\left(x_{\tau(n)}\right)-w\right\|^{2} / \beta_{\tau(n)} \rightarrow 0$ and $2 \alpha_{\tau(n)}(1-$ $\left.\alpha_{\tau(n)}\right) / \beta_{\tau(n)} \rightarrow 1 /(1-\theta)$. Therefore, we obtain

$$
\limsup _{n \rightarrow \infty} \frac{\gamma_{\tau(n)}}{\beta_{\tau(n)}} \leq 0
$$

On the other hand, from (3.3) and (3.6), we know that

$$
\begin{aligned}
\left\|x_{\tau(n)+1}-w\right\|^{2} & \leq\left(1-\beta_{\tau(n)}\right)\left\|x_{\tau(n)}-w\right\|^{2}+\gamma_{\tau(n)} \\
& \leq\left(1-\beta_{\tau(n)}\right)\left\|x_{\tau(n)+1}-w\right\|^{2}+\gamma_{\tau(n)}
\end{aligned}
$$

for every $n \in \mathbb{N}$. Thus, by $\beta_{\tau(n)}>0$, this shows that

$$
\left\|x_{\tau(n)+1}-w\right\|^{2} \leq \frac{\gamma_{\tau(n)}}{\beta_{\tau(n)}}
$$

for every $n \in \mathbb{N}$.

Finally, we obtain a contradiction that $\left\|x_{n}-w\right\| \rightarrow 0$. Using (3.7), (3.9), and (3.8), we conclude that

$$
\limsup _{n \rightarrow \infty}\left\|x_{n}-w\right\|^{2} \leq \limsup _{n \rightarrow \infty}\left\|x_{\tau(n)+1}-w\right\|^{2} \leq \limsup _{n \rightarrow \infty} \frac{\gamma_{\tau(n)}}{\beta_{\tau(n)}} \leq 0,
$$

and hence $\left\|x_{n}-w\right\| \rightarrow 0$, which is a contradiction. 
Proof of Theorem 3.1 We first show that $S_{n} x_{n}-x_{n} \rightarrow 0$. Since $S_{n}$ is quasinonexpansive, it follows from (3.2) that

$$
0 \leq\left\|x_{n}-w\right\|-\left\|S_{n} x_{n}-w\right\| \leq\left\|x_{n}-w\right\|-\left\|x_{n+1}-w\right\|+\alpha_{n}\left\|f_{n}\left(x_{n}\right)-w\right\|
$$

for every $n \in \mathbb{N}$, so that $\left\|x_{n}-w\right\|-\left\|S_{n} x_{n}-w\right\| \rightarrow 0$ by Lemma 3.4, $\alpha_{n} \rightarrow 0$, and Lemma 3.2. Since $\left\{S_{n}\right\}$ is strongly quasinonexpansive type and $\left\{x_{n}\right\}$ is bounded, we conclude that $S_{n} x_{n}-$ $x_{n} \rightarrow 0$.

Since $\left\{S_{n}\right\}$ satisfies the condition (Z), Lemma 2.8 implies that

$$
\limsup _{n \rightarrow \infty}\left\langle S_{n} x_{n}-w, f_{1}(w)-w\right\rangle \leq 0
$$

This shows that $\lim _{\sup _{n \rightarrow \infty}} \gamma_{n} / \beta_{n} \leq 0$ by using Lemmas 3.2 and 3.3. On the other hand, it follows from (3.3) that

$$
\left\|x_{n+1}-w\right\|^{2} \leq\left(1-\beta_{n}\right)\left\|x_{n}-w\right\|^{2}+\beta_{n} \frac{\gamma_{n}}{\beta_{n}}
$$

for every $n \in \mathbb{N}$. Therefore, noting that $\sum_{n=1}^{\infty} \beta_{n}=\infty$ and using Lemma 2.9, we conclude that $x_{n}-w \rightarrow 0$.

A direct consequence of Theorem 3.1 is the following corollary, which is a slight generalization of [8, Theorem 2.3].

Corollary 3.5 Let $H$ be a real Hilbert space, $C$ a nonempty closed convex subset of $H$, $S: C \rightarrow C$ a strongly quasinonexpansive mapping, $\left\{\alpha_{n}\right\}$ a sequence in $(0,1]$ such that $\alpha_{n} \rightarrow$ 0 and $\sum_{n=1}^{\infty} \alpha_{n}=\infty$, and $f: C \rightarrow C$ a $\theta$-contraction with respect to $F=\operatorname{Fix}(S)$, where $0 \leq$ $\theta<1$. Let $\left\{x_{n}\right\}$ be a sequence defined by $x_{1} \in C$ and

$$
x_{n+1}=\alpha_{n} f\left(x_{n}\right)+\left(1-\alpha_{n}\right) S x_{n}
$$

for $n \in \mathbb{N}$. Suppose that $I-S$ is demiclosed at 0 . Then $\left\{x_{n}\right\}$ converges strongly to $w \in F$, where $w$ is the unique fixed point of a contraction $P_{F} \circ f$.

Proof Set $S_{n}=S$ and $f_{n}=f$ for $n \in \mathbb{N}$. Then it is clear that $\bigcap_{n=1}^{\infty} \operatorname{Fix}\left(S_{n}\right)=\operatorname{Fix}(S),\left\{S_{n}\right\}$ is strongly quasinonexpansive type, $\left\{S_{n}\right\}$ satisfies the condition $(\mathrm{Z})$, and $\left\{f_{n}\right\}$ is stable on $C$. Thus Theorem 3.1 implies the conclusion.

\section{Application to a variational inequality problem}

In this section, applying Theorem 3.1, we study an approximation method for the following variational inequality problem.

Problem 4.1 Let $\kappa$ and $\eta$ be positive real numbers such that $\eta^{2}<2 \kappa$. Let $F$ be a nonempty closed convex subset of $H$ and $A: H \rightarrow H$ a $\kappa$-strongly monotone and $\eta$-Lipschitz continuous mapping, that is, we assume that $\langle x-y, A x-A y\rangle \geq \kappa\|x-y\|^{2}$ and $\|A x-A y\| \leq \eta\|x-y\|$ for all $x, y \in H$. Then find $z \in F$ such that

$$
\langle y-z, A z\rangle \geq 0 \quad \text { for all } y \in F .
$$


The solution set of Problem 4.1 is denoted by $\operatorname{VI}(F, A)$. Under the assumptions of Problem 4.1, it is known that the following hold; see, for example, [22].

- $\kappa \leq \eta, 0 \leq 1-2 \kappa+\eta^{2}<1$ and $I-A$ is a $\theta$-contraction, where $\theta=\sqrt{1-2 \kappa+\eta^{2}}$;

- Problem 4.1 has a unique solution and $\operatorname{VI}(F, A)=\operatorname{Fix}\left(P_{F}(I-A)\right)$.

Remark 4.2 The assumption that $\eta^{2}<2 \kappa$ in Problem 4.1 is not restrictive. Indeed, let $F$ be a nonempty closed convex subset of $H$ and $\tilde{A}$ a $\tilde{\kappa}$-strongly monotone and $\tilde{\eta}$-Lipschitz continuous mapping, where $\tilde{\kappa}>0$ and $\tilde{\eta}>0$. Set $A=\mu \tilde{A}, \kappa=\mu \tilde{\kappa}$, and $\eta=\mu \tilde{\eta}$, where $\mu$ is a positive constant such that $\mu \tilde{\eta}^{2}<2 \tilde{\kappa}$. Then it is easy to verify that $A$ is $\kappa$-strongly monotone and $\eta$-Lipschitz continuous, $\eta^{2}<2 \kappa$, and moreover, $\operatorname{VI}(F, A)=\operatorname{VI}(F, \tilde{A})$.

Using Theorem 3.1, we obtain the following convergence theorem for Problem 4.1.

Theorem 4.3 Let $H, \kappa, \eta$, and $A$ be the same as in Problem 4.1. Let $\left\{S_{n}\right\}$ be a sequence of mappings of $H$ into $H$ such that $F=\bigcap_{n=1}^{\infty} \operatorname{Fix}\left(S_{n}\right)$ is nonempty, and $\left\{\alpha_{n}\right\}$ the same as in Theorem 3.1. Let $\left\{x_{n}\right\}$ be a sequence defined by $x_{1} \in H$ and

$$
x_{n+1}=S_{n} x_{n}-\alpha_{n} A S_{n} x_{n}
$$

for $n \in \mathbb{N}$. Suppose that $\left\{S_{n}\right\}$ is strongly quasinonexpansive type and $\left\{S_{n}\right\}$ satisfies the condition (Z). Then $\left\{x_{n}\right\}$ converges strongly to the unique solution of Problem 4.1.

Proof Set $f_{n}=(I-A) S_{n}$ for $n \in \mathbb{N}$ and $\theta=\sqrt{1-2 \kappa+\eta^{2}}$. Since $I-A$ is a $\theta$-contraction and $S_{n}$ is quasinonexpansive, Lemma 2.2 implies that each $f_{n}$ is a $\theta$-contraction with respect to $F$. It is obvious that $\left\{f_{n}\right\}$ is stable on $F$. Moreover, it follows from (4.1) that

$$
x_{n+1}=\alpha_{n} f_{n}\left(x_{n}\right)+\left(1-\alpha_{n}\right) S_{n} x_{n}
$$

for $n \in \mathbb{N}$. Thus Theorem 3.1 implies that $\left\{x_{n}\right\}$ converges strongly to $w=\left(P_{F} \circ f_{1}\right)(w)=$ $P_{F}(I-A) w$, which is the unique solution of Problem 4.1.

Remark 4.4 The iteration (4.1) is called the hybrid steepest descent method; see $[9,10]$ for more details.

We finally construct an example of $\left\{S_{n}\right\}$ in Theorem 4.3 by using the notion of a subgradient projection.

Let $g: H \rightarrow \mathbb{R}$ be a continuous and convex function such that

$$
C=\{x \in H: g(x) \leq 0\}
$$

is nonempty and $h: H \rightarrow H$ a mapping such that $h(x) \in \partial g(x)$ for all $x \in H$, where $\partial g$ denotes the subdifferential mapping of $g$ defined by

$$
\partial g(x)=\{z \in H: g(x)+\langle y-x, z\rangle \leq g(y)(\forall y \in H)\}
$$

for all $x \in H$. Then the subgradient projection $P_{g, h}: H \rightarrow H$ with respect to $g$ and $h$ is defined by $P_{g, h} x=P_{L(x)} x$ for all $x \in H$, where $P_{L(x)}$ denotes the metric projection of $H$ onto 
the set $L(x)$ defined by

$$
L(x)=\{y \in H: g(x)+\langle y-x, h(x)\rangle \leq 0\}
$$

for all $x \in H$. Note that $C$ is a subset of $L(x)$ for all $x \in H$ and that $L(x)$ is a closed half space for all $x \in H \backslash C$. According to [23, Section 7], [24, Proposition 2.3], and [25, Proposition 1.1.11], we know the following:

(S1) $\operatorname{Fix}\left(P_{g, h}\right)=C$;

(S2) $\left\langle z-P_{g, h} x, x-P_{g, h} x\right\rangle \leq 0$ for all $z \in C$ and $x \in H$;

(S3) if $g(V)$ is bounded for each bounded subset $V$ of $H$, then $I-P_{g, h}$ is demiclosed at 0 .

It is known that the metric projection $P_{D}$ of $H$ onto a nonempty closed convex subset $D$ of $H$ coincides with the subgradient projection $P_{g, h}$ with respect to $g$ and $h$ defined by $g(x)=\inf _{y \in D}\|x-y\|$ for all $x \in H$ and

$$
h(x)= \begin{cases}0 & (x \in D) ; \\ \left(x-P_{D} x\right) /\left\|x-P_{D} x\right\| & (x \in H \backslash D) .\end{cases}
$$

The subgradient projection is not necessarily nonexpansive. In fact, if $g: \mathbb{R} \rightarrow \mathbb{R}$ and $h: \mathbb{R} \rightarrow \mathbb{R}$ are defined by $g(x)=\max \{x, 2 x-1\}$ for all $x \in \mathbb{R}$ and $h(x)=1$ if $x<1 ; h(x)=2$ if $x \geq 1$, then $P_{g, h}$ is given by

$$
P_{g, h}(x)= \begin{cases}x & (x \leq 0) \\ 0 & (0<x<1) \\ 1 / 2 & (x \geq 1)\end{cases}
$$

and is not nonexpansive.

Using (S1), (S2), and (S3), we show the following.

Example 4.5 Let $g: H \rightarrow \mathbb{R}$ be a continuous and convex function such that $C=\{x \in H$ : $g(x) \leq 0\}$ is nonempty and $g(V)$ is bounded for each bounded subset $V$ of $H, h: H \rightarrow H$ a mapping such that $h(x) \in \partial g(x)$ for all $x \in H$, and $\left\{S_{n}\right\}$ a sequence of mappings of $H$ into $H$ defined by

$$
S_{n}=\beta_{n} I+\left(1-\beta_{n}\right) P_{g, h}
$$

for all $n \in \mathbb{N}$, where $\left\{\beta_{n}\right\}$ is a sequence of real numbers such that $-1<\inf _{n} \beta_{n}$ and $\sup _{n} \beta_{n}<1$. Then the following hold:

(i) $\operatorname{Fix}\left(S_{n}\right)=C$ for all $n \in \mathbb{N}$;

(ii) $\left\{S_{n}\right\}$ is strongly quasinonexpansive type;

(iii) $\left\{S_{n}\right\}$ satisfies the condition (Z).

Proof Since $\beta_{n} \neq 1$ for all $n \in \mathbb{N}$, the part (i) obviously follows from (S1).

We first show (ii). By (i), we know that $\bigcap_{n=1}^{\infty} \operatorname{Fix}\left(S_{n}\right)=C$ is nonempty. Let $n \in \mathbb{N}, p \in C$, and $x \in H$ be given. Then we have

$$
\begin{aligned}
\left\|S_{n} x-p\right\|^{2}+\left\|x-S_{n} x\right\|^{2}-\|x-p\|^{2} & =2\left\langle S_{n} x-x, S_{n} x-p\right\rangle \\
& =2\left(1-\beta_{n}\right)\left\langle p-S_{n} x, x-P_{g, h} x\right\rangle .
\end{aligned}
$$


It follows from (S2) that

$$
\left\langle p-S_{n} x, x-P_{g, h} x\right\rangle \leq\left\langle P_{g, h} x-S_{n} x, x-P_{g, h} x\right\rangle .
$$

On the other hand, we also know that

$$
\begin{aligned}
& \left\langle P_{g, h} x-S_{n} x, x-P_{g, h} x\right\rangle \\
& \quad=-\left\|P_{g, h} x-x\right\|^{2}+\left\langle x-S_{n} x, x-P_{g, h} x\right\rangle \\
& \quad \leq-\left(\left\|P_{g, h} x-x\right\|-\frac{1}{2}\left\|x-S_{n} x\right\|\right)^{2}+\frac{1}{4}\left\|x-S_{n} x\right\|^{2} \leq \frac{1}{4}\left\|x-S_{n} x\right\|^{2} .
\end{aligned}
$$

By (4.2), (4.3), and (4.4), each $S_{n}$ satisfies

$$
\left\|S_{n} x-p\right\|^{2}+\frac{1}{2}\left(1+\beta_{n}\right)\left\|x-S_{n} x\right\|^{2} \leq\|x-p\|^{2}
$$

for all $p \in C$ and $x \in H$. Since $\left(1+\beta_{n}\right) / 2>0$, we know that each $S_{n}$ is quasinonexpansive.

Let $\left\{x_{n}\right\}$ be a bounded sequence in $H$ such that $\left\|x_{n}-p\right\|-\left\|S_{n} x_{n}-p\right\| \rightarrow 0$ for some $p \in C$. Since $\left\{S_{n} x_{n}\right\}$ is bounded, it follows from (4.5) that

$$
\frac{1}{2}\left(1+\beta_{n}\right)\left\|x_{n}-S_{n} x_{n}\right\|^{2} \leq\left\|x_{n}-p\right\|^{2}-\left\|S_{n} x_{n}-p\right\|^{2} \rightarrow 0
$$

and hence $S_{n} x_{n}-x_{n} \rightarrow 0$ by $\inf _{n}\left(1+\beta_{n}\right)>0$. Thus $\left\{S_{n}\right\}$ is strongly quasinonexpansive type.

We finally show (iii). Let $\left\{y_{n}\right\}$ be a bounded sequence in $H$ such that $S_{n} y_{n}-y_{n} \rightarrow 0$. By the definition of $S_{n}$, we have

$$
\left\|P_{g, h} y_{n}-y_{n}\right\|=\frac{1}{1-\beta_{n}}\left\|S_{n} y_{n}-y_{n}\right\|
$$

for all $n \in \mathbb{N}$. Since $\inf _{n}\left(1-\beta_{n}\right)>0$, we obtain $P_{g, h} y_{n}-y_{n} \rightarrow 0$. Consequently, by (S1) and (S3), we know that $\left\{S_{n}\right\}$ satisfies the condition $(\mathrm{Z})$.

\section{Competing interests}

The authors declare that they have no competing interests.

Authors' contributions

All authors read and approved the final manuscript.

\section{Author details}

'Department of Economics, Chiba University, Yayoi-cho, Inage-ku, Chiba-shi, Chiba, 263-8522, Japan. ${ }^{2}$ Department of Computer Science and Intelligent Systems, Oita University, Dannoharu, Oita-shi, Oita, 870-1192, Japan.

\section{Acknowledgements}

This paper is dedicated to Professor Wataru Takahashi on the occasion of his 70th birthday.

Received: 27 September 2013 Accepted: 18 December 2013 Published: 22 Jan 2014

\section{References}

1. Moudafi, A: Viscosity approximation methods for fixed-points problems. J. Math. Anal. Appl. 241, 46-55 (2000)

2. Aoyama, K, Kimura, Y, Kohsaka, F: Strong convergence theorems for strongly relatively nonexpansive sequences and applications. J. Nonlinear Anal. Optim. 3, 67-77 (2012)

3. Xu, H-K: Viscosity approximation methods for nonexpansive mappings. J. Math. Anal. Appl. 298, 279-291 (2004)

4. Suzuki, T: Moudafi's viscosity approximations with Meir-Keeler contractions. J. Math. Anal. Appl. 325, 342-352 (2007) 
5. Aoyama, K, Kimura, Y: Viscosity approximation methods with a sequence of contractions. CUBO (to appear)

6. Halpern, B: Fixed points of nonexpanding maps. Bull. Am. Math. Soc. 73, 957-961 (1967)

7. Maingé, P-E: The viscosity approximation process for quasi-nonexpansive mappings in Hilbert spaces. Comput. Math. Appl. 59, 74-79 (2010)

8. Wongchan, K, Saejung, S: On the strong convergence of viscosity approximation process for quasinonexpansive mappings in Hilbert spaces. Abstr. Appl. Anal. 2011, Article ID 385843 (2011)

9. Yamada, I, Ogura, N: Hybrid steepest descent method for variational inequality problem over the fixed point set of certain quasi-nonexpansive mappings. Numer. Funct. Anal. Optim. 25, 619-655 (2004)

10. Cegielski, A, Zalas, R: Methods for variational inequality problem over the intersection of fixed point sets of quasi-nonexpansive operators. Numer. Funct. Anal. Optim. 34, 255-283 (2013)

11. Dotson, WG Jr.: Fixed points of quasi-nonexpansive mappings. J. Aust. Math. Soc. 13, 167-170 (1972)

12. Takahashi, W: Introduction to Nonlinear and Convex Analysis. Yokohama Publishers, Yokohama (2009)

13. Maingé, P-E: Strong convergence of projected subgradient methods for nonsmooth and nonstrictly convex minimization. Set-Valued Anal. 16, 899-912 (2008)

14. Aoyama, K: Asymptotic fixed points of sequences of quasi-nonexpansive type mappings. In: Banach and Function Spaces III, pp. 343-350. Yokohama Publishers, Yokohama (2011)

15. Aoyama, K, Kimura, Y: Strong convergence theorems for strongly nonexpansive sequences. Appl. Math. Comput. 217, 7537-7545 (2011)

16. Aoyama, K, Kohsaka, F, Takahashi, W: Strong convergence theorems by shrinking and hybrid projection methods for relatively nonexpansive mappings in Banach spaces. In: Nonlinear Analysis and Convex Analysis, pp. 7-26. Yokohama Publishers, Yokohama (2009)

17. Aoyama, K, Kohsaka, F, Takahashi, W: Strongly relatively nonexpansive sequences in Banach spaces and applications. J. Fixed Point Theory Appl. 5, 201-224 (2009)

18. Aoyama, K, Kimura, Y, Takahashi, W, Toyoda, M: On a strongly nonexpansive sequence in Hilbert spaces. J. Nonlinear Convex Anal. 8, 471-489 (2007)

19. Aoyama, K, Kimura, Y, Takahashi, W, Toyoda, M: Strongly nonexpansive sequences and their applications in Banach spaces. In: Fixed Point Theory and Its Applications, pp. 1-18. Yokohama Publishers, Yokohama (2008)

20. Xu, H-K: Iterative algorithms for nonlinear operators. J. Lond. Math. Soc. (2) 66, 240-256 (2002)

21. Aoyama, K, Kimura, Y, Takahashi, W, Toyoda, M: Approximation of common fixed points of a countable family of nonexpansive mappings in a Banach space. Nonlinear Anal. 67, 2350-2360 (2007)

22. Aoyama, K, Kimura, Y: A note on the hybrid steepest descent methods. arXiv:1101.0881 [math.FA]

23. Bauschke, HH, Borwein, JM: On projection algorithms for solving convex feasibility problems. SIAM Rev. 38, 367-426 (1996)

24. Bauschke, HH, Combettes, PL: A weak-to-strong convergence principle for Fejér-monotone methods in Hilbert spaces. Math. Oper. Res. 26, 248-264 (2001)

25. Butnariu, D, lusem, AN: Totally Convex Functions for Fixed Points Computation and Infinite Dimensional Optimization. Applied Optimization, vol. 40. Kluwer Academic, Dordrecht (2000)

10.1186/1687-1812-2014-17

Cite this article as: Aoyama and Kohsaka: Viscosity approximation process for a sequence of quasinonexpansive mappings. Fixed Point Theory and Applications 2014, 2014:17

\section{Submit your manuscript to a SpringerOpen ${ }^{\ominus}$ journal and benefit from:}

- Convenient online submission

Rigorous peer review

- Immediate publication on acceptance

Open access: articles freely available online

- High visibility within the field

- Retaining the copyright to your article 